\title{
Diseño de una guía metodológica para la implementación del sistema integral de gestión y control de la Gobernación de Cundinamarca*
}

\author{
Design of a methodological guide for the implementation \\ of the integrated management and control of the Government \\ of Cundinamarca
}

Recibido: 4 de abril de 2016 Revisado: 11 de abril de 2016 Aceptado: 13 de junio de 2016

Claudia Patricia Lópes Cabrera*

Secretaría de Salud de Cundinamarca

Víctor Hugo Velásques Ortis ***

Gobernación de Cundinamarca

\section{RESUMEN}

La investigación está dirigida a diseñar una guía metodológica para la implementación del sistema integral de gestión y control de la Gobernación de Cundinamarca, certificada en NTG-ISO 9001:2008 y NTC-GP 1000:2009 y busca dar respuesta a la pregunta: ¿Con qué metodología se puede lograr la implementación de los sistemas de gestión en la Gobernación de Cundinamarca? La investigación, de diseño mixto de triangulación concurrente fundamentada en Karapetrovic y Willborn (1998) y Rocha y Karapetrovic (2008) evaluó el sistema existente, la percepción del cliente interno, identificó metodologías

\footnotetext{
Artículo de resultado de investigación.

** Odontóloga. Especialista en Administración y Gerencia de Sistemas de la Calidad. Magíster (c) en Calidad y Gestión Integral. Profesional especializado en la Secretaría de Salud de Cundinamarca. Correo electrónico: sogcas@gmail.com

*** Ingeniero industrial. Especialista en Administración y Gerencia de Sistemas de la Calidad. Magíster (c) en Calidad y Gestión Integral. Director de desarrollo Organizacional en la Gobernación de Cundinamarca. Correo electrónico: vhvortiz@yahoo.com
} 
utilizadas en otras entidades y correlacionó guías metodológicas aplicando encuestas y entrevistas. Se encuentran la necesidad de articular el sistema integrado de gestión con el Plan Departamental de Desarrollo, así como una relación inversamente proporcional entre conocimiento/apropiación y la percepción. La guía elaborada conjuga la teoría del Círculo de Oro de Simón Sinek, el PHVA y la estructura de alto nivel, orientando la implementación en forma sencilla, clara, de fácil lectura, acompañándola de un anexo lúdico.

Palabras clave: gestión de la calidad, gestión pública, gestión integral, sistema integrado de gestión.

\section{ABSTRACT}

Research aimed at designing a methodological guide for the implementation of the integrated management and control of Cundinamarca Governorship, certified NTC-ISO 9001: 2008 and NTC GP 1000: 2009, seeks to answer the question: What can be active methodology achieve the implementation of management systems in Cundinamarca? Research, mixed design of concurrent triangulation based on Karapetrovic, Willborn (1998) and Rocha and Karapetrovic (2015), evaluated the existing system, internal customer perception, identify methodologies used in other entities, correlated methodological guidelines; using surveys and interviews. Finding the need to link it with the Departmental Development Plan; and the inverse relationship between knowledge/ownership and perception. The elaborate theory combines Guide gold circle Simon Sinek, the PDCA and the high-level structure, guiding the implementation, clear, easily readable, accompanied by a playful Annex.

Keywords: Quality management, public management, integrated management system, government policy and regulation.

\section{INTRODUCCIÓN}

La Ley 872 de 2003 (Congreso de la República de Colombia, 2003), establece la obligatoriedad de implementar el sistema de gestión de la calidad en entidades del Estado, para mejorar su desempeño y satisfacer las necesidades de la comunidad a la cual dirigen sus acciones, así mismo otras normas exigen implementar el Modelo Estándar de Control Interno y el sistema de desarrollo administrativo, soportado en el Modelo Integrado de Planeación y Gestión, orientando a las entidades del sector público a contar con sistemas integrales de gestión y control, lo que es un reto por las características del sector, los continuos cambios normativos y la rotación del talento humano.

A la Gobernación de Cundinamarca, certificada con NTC-ISO 9001:2008 (Instituto Colombiano de Normas Técnicas y Certificación, 2008) y NTC-GP 1000:2009, le corresponde implementar los sistemas/modelos descritos, contando actualmente con un sistema integral de gestión y control del que no hacen parte todos los sistemas obligatorios que son de su competencia, otros se implementan de forma independiente, como es el caso del de seguridad y salud en el trabajo exigido por el Decreto 1072 de 2015, y otros voluntarios como el sistema de gestión ambiental, entre otros, sin contar con una metodología que oriente la implementación, lo cual lleva a plantear como problema de investigación: ¿Con qué metodología se puede lograr la implementación de los sistemas de gestión en la Gobernación de Cundinamarca? Contemplando como preguntas de sistematización: ¿El conocimiento del sistema integral de gestión y control, por parte del cliente interno, es coherente con la percepción que este tiene sobre su conocimiento y utilidad? ¿El sistema integral de gestión y control está implementado por procesos o por estructura funcional? ¿Se conocen y aplican metodologías de implementación de sistemas de gestión en otras gobernaciones y en la Alcaldía Mayor de Bogotá? 
Por lo anteriormente expuesto la investigación es necesaria, por cuanto la gobernación requiere de una guía que oriente la implementación del sistema integral de gestión y control, a pesar de contar con un sistema implementado y estar certificada, por la multiplicidad de sistemas de gestión por mandato legal, como es el caso, entre otros, del sistema de gestión de la calidad para entidades del Estado (Congreso de la República de Colombia, 2003), el sistema de desarrollo administrativo (Congreso de la República de Colombia, 1998) y el Modelo Estándar de Control Interno (Departamento Administrativo de la Función Pública, 2014) o de forma voluntaria debe o decide implementar, y por el alto número de entidades que la integran (Gobernación de Cundinamarca, 2015), bajo una estructura funcional de secretarías, unidades administrativas y oficinas, conformadas por más de 1.500 servidores públicos de diferentes niveles y procesos (Departamento Administrativo de la Función Pública, 2005).

Adicionalmente, la Gobernación de Cundinamarca requiere contar con una guía metodológica que le permita articular los diferentes sistemas de gestión con el Plan Departamental de Desarrollo, disminuyendo reprocesos, optimizando recursos y tiempos de implementación, permitiéndole al cliente interno comprender el propósito del sistema integral de gestión y control y apropiarse del mismo, y adicionalmente para contribuir al logro de las metas y objetivos del Plan Departamental de Desarrollo en beneficio de los 116 municipios del departamento.

Es así como la implementación del sistema integral de gestión y control de la gobernación es una obligación por mandato legal, nacional y departamental (Gobernación de Cundinamarca, 2008), y es un reto, dado que el Sistema de Gestión Integral posibilita y simplifica la implantación de un único sistema de gestión eficaz y adecuado para la empresa (Fraguela et al., 2011), lo cual es factible de alcanzar con una guía metodológica que oriente el proceso.
Por otra parte, es necesario contemplar que la guía aporta al cumplimiento de la responsabilidad que recae sobre el gobernador del departamento, como máxima autoridad, de desarrollar, implementar, mantener, revisar y perfeccionar el sistema de gestión de la calidad de forma complementaria con otros sistemas, ya que su incumplimiento genera una causal de mala conducta, de acuerdo con lo establecido en la Ley 872 de 2003 (Congreso de la República de Colombia, 2003).

En el componente teórico y conceptual la investigación se sustenta en los planteamientos de Karapetrovic y Willborn (1998) y Rocha y Karapetrovic (2015), cuyo aporte se centra en abordar el concepto de sistema y sistema de sistemas; definiéndolo como un sistema formado por subsistemas de función específica que pierden completamente sus identidades únicas para ser un "sistema de sistemas". En este enfoque sistémico, cada sistema aporta al otro, requiere del otro y se fortalecen mutuamente, sus partes se correlacionan y guardan interdependencia. Es un pensamiento sistémico fundamental en la etapa de planeación y que durante el desarrollo de la investigación se enriquece aún más con los aportes de Fritjof Capra (1998), físico teórico austriaco, quien aborda el tema en su obra $L a$ trama de la vida.

En cuanto al concepto de Sistema Integral de Gestión (SIG), Rocha y Karapetrovic (2015) lo definen como una alternativa que permite vincular nuevos sistemas con los ya existentes o comenzar con un esquema integrado desde el inicio, mitigando los problemas de aislamiento, desconexión y bajo desempeño entre sistemas, de allí la importancia de la integralidad. En el modelo de "enfoque de sistemas", desarrollado por Karapetrovic y Willborn y ajustado por Jonker y Karapetrovic en el 2004, el término integración es definido como la interdependencia de los elementos del sistema y el término alineación es considerado un sustituto de la palabra integración. 
La integración de sistemas es justificada adicionalmente por Rocha y Karapetrovic (2008) debido a que:

Tener uno o más sistemas de gestión aislados dentro de la organización dificulta significativamente incrementar la productividad y efectividad de los mismos y de la empresa. Al estar aislados cada uno de los sistemas implementados demanda recursos individuales (afectando la productividad) y puede generar conflictos de objetivos (afectando la efectividad) (p. 1).

Las hipótesis son las siguientes: la Gobernación de Cundinamarca ha implementado el sistema integral de gestión y control sin una metodología específica, las gobernaciones del país y la Alcaldía Mayor han utilizado la metodología del DAPF para la implementación de sus sistemas de gestión y el conocimiento y apropiación del cliente interno sobre el Sistema Integral de Gestión, el cual es directamente proporcional a la forma como lo percibe. Conduciendo al objetivo principal de la investigación: diseñar una guía metodológica para la implementación del sistema integral de gestión y control de la Gobernación de Cundinamarca, contribuyendo a fortalecer la gestión institucional y la apropiación del mismo por parte de los clientes internos; $\mathrm{y}$ a los objetivos específicos: 1) identificar las fortalezas y oportunidades de mejora del sistema integral de gestión y control de la Gobernación de Cundinamarca; 2) evaluar la apropiación y conocimiento del sistema integral de gestión y control por parte del cliente interno en los diferentes procesos y niveles jerárquicos; 3) correlacionar algunas metodologías de implementación de sistemas de gestión existentes en el contexto nacional e internacional; 4) identificar los sistemas implementados en gobernaciones y en la Alcaldía Mayor de Bogotá, metodologías utilizadas, dificultades y expectativas frente a una guía metodológica; 5) validar la guía metodológica diseñada a través de un panel de expertos; 6) presentar la iniciativa de publicación de la guía metodológica elaborada como un aporte de la Gobernación de Cundinamarca al sector público.

\section{METODOLOGÍA}

El proceso metodológico seguido consta de nueve momentos de investigación, como se observa en la siguiente figura:

Figura 1. Momentos de investigación.

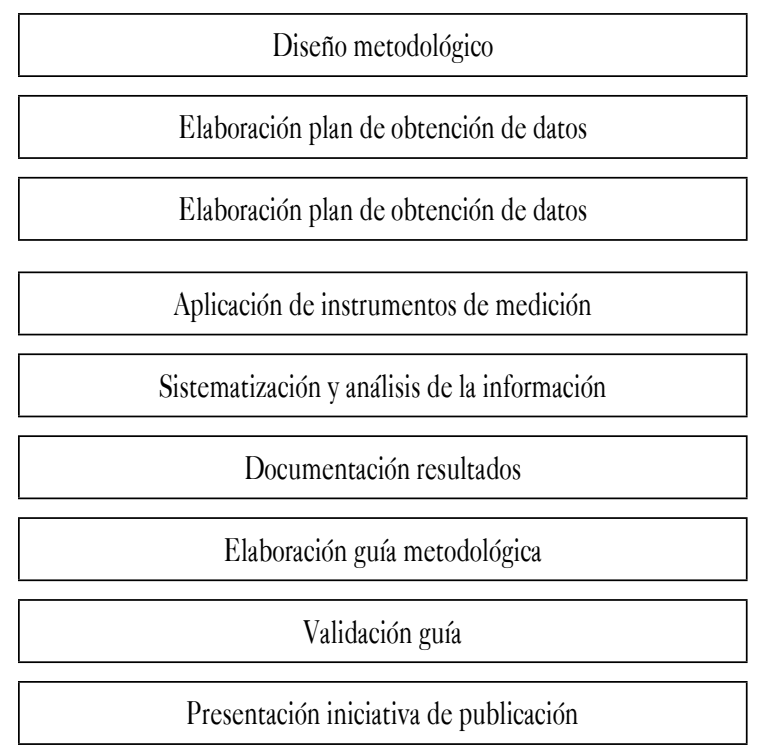

Fuente: elaboración propia.

La investigación se desarrolló con un diseño mixto de triangulación concurrente, con el fin de aplicar en paralelo los diferentes instrumentos de medición para aprovechar las ventajas cualitativas y cuantitativas de cada método, y así minimizar sus debilidades (Hernández et al., 2014, pp. 532-554).

Con base en lo anterior se recolectó y analizó la información requerida, de forma paralela, partiendo de los datos cuantitativos y cualitativos. La ventaja de este diseño, según Hernández et al. (2014), "es que puede otorgar validez cruzada o de criterio y pruebas a estos últimos, además de que normalmente requiere menor tiempo de implementación" (p. 557), lo cual era necesario por encontrarse la gobernación terminando el periodo legal constitucional establecido. En la figura 2 se encuentra esquematizado el diseño de la investigación. 
Figura 2. Diseño mixto de triangulación concurrente.

\section{CUALITATIVO}

- Correlación guías metodológicas

- Identificación fortaleza y oportunidades de mejora SIGC

- Entrevistas semiestructuradas en profundidad

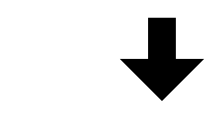

\section{RESULTADOS}

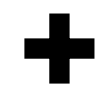

CUANTITATIVO

- Encuesta cliente interno

- Encuesta de conocimiento y aplicación a gobernaciones y en la Alcaldía Mayor de Bogotá

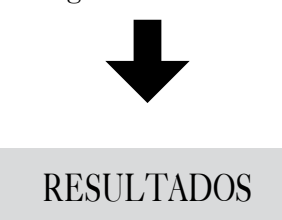

Fuente: elaboración propia.

Una vez obtenidos los resultados cuantitativos y los cualitativos se analizaron e interpretaron obteniendo inferencias cualitativas-cuantitativas y metainferencias de investigación, las cuales son base para la elaboración de la guía metodológica resultante de la investigación.
El diseño muestral se desarrolló con apoyo de la Facultad de Estadística de la Universidad Santo Tomás, en cuanto a encuestas a aplicar a clientes internos, a las gobernaciones y en la Alcaldía Mayor de Bogotá, en tanto se definió aplicar las entrevistas al 100 $\%$ del talento humano de la dirección de desarrollo organizacional. En la tabla 1 se presenta la muestra y la determinación del diseño muestral.

Tabla 1. Muestra y determinación diseño muestral.

\begin{tabular}{|l|l|l|l|}
\hline \multicolumn{1}{|l|}{ Instrumento de medición } & \multicolumn{1}{|c|}{ Universo } & \multicolumn{1}{|c|}{ Muestra } & \multicolumn{1}{c|}{ Diseño Muestral } \\
\hline $\begin{array}{l}\text { Encuesta a clientes internos } \\
\text { de la gobernación. }\end{array}$ & $\begin{array}{l}1.520 \\
\text { Funcionarios en planta de } \\
\text { personal. }\end{array}$ & $\begin{array}{l}734 \text { funcionarios en planta de } \\
\text { personal. }\end{array}$ & $\begin{array}{l}\text { Muestreo bietápico partiendo de los procesos, } \\
\text { y de ellos a las secretarías. En la primera etapa } \\
\text { se hizo un estratificado MAS y en la segunda un } \\
\text { conglomerado. }\end{array}$ \\
\hline $\begin{array}{l}\text { Encuesta a gobernaciones. } \\
\text { 32 gobernaciones y 1 } \\
\text { Alcaldía Mayor. }\end{array}$ & $\begin{array}{l}32 \text { gobernaciones y 1 Alcaldía } \\
\text { Mayor. }\end{array}$ & $\begin{array}{l}\text { Diseño probabilístico estratificado para } \\
\text { determinar las gobernaciones por región (primera } \\
\text { etapa). Diseño de inclusión forzosa incluyendo } \\
\text { las 5 gobernaciones con sistema de gestión } \\
\text { certificado. }\end{array}$ \\
\hline $\begin{array}{l}\text { Entrevista a funcionarios de } \\
\text { planta de la dependencia a } \\
\text { cargo del sistema integral } \\
\text { de gestión y control de la } \\
\text { gobernación. }\end{array}$ & $\begin{array}{l}7 \text { funcionarios de } \\
\text { dirección de desarrollo } \\
\text { organizacional. }\end{array}$ & $\begin{array}{l}7 \text { funcionarios de dirección de } \\
\text { desarrollo organizacional. }\end{array}$ & Universo. \\
\hline
\end{tabular}

Fuente: elaboración propia. 
En el desarrollo de la investigación se determinaron los instrumentos de medición requeridos para recolectar la información de forma organizada y clasificada. Los instrumentos parten de la conceptualización de medición como "el proceso de vincular conceptos abstractos con indicadores empíricos" (Hernández et al., 2014, p. 534), de manera que se establecen las fuentes y técnicas para obtener los datos.

Los instrumentos de medición definidos para el componente cualitativo se relacionan a continuación: matriz de identificación de fortalezas y oportunidades de mejora del sistema integral de gestión y control de la gobernación, matriz de correlación guías metodológicas, entrevista semiestructurada a profundidad aplicadas a clientes internos de la dirección de desarrollo organizacional de la Secretaría de la Función Pública, dependencia a cargo del sistema integral de gestión y control de la gobernación. Para el componente cuantitativo, se definieron los siguientes instrumentos de medición: encuesta a clientes internos de la gobernación, encuesta a gobernaciones y a la Alcaldía Mayor de Bogotá.

Una vez definidos los datos requeridos, se procedió a diseñar los instrumentos de medición necesarios, para ello previamente se relacionó cada instrumento con el objetivo específico de investigación al que responde, la muestra 0 el universo al que se le aplicaría el instrumento de medición y la técnica de sistematización a emplear. En la siguiente figura se aprecia lo anteriormente descrito:

Figura 3. Relación de instrumentos y técnicas con objetivos específicos.

\section{INSTRUMENTOS Y TECNICAS DE INVESTIGACIÓN}

CUANTITATIVO

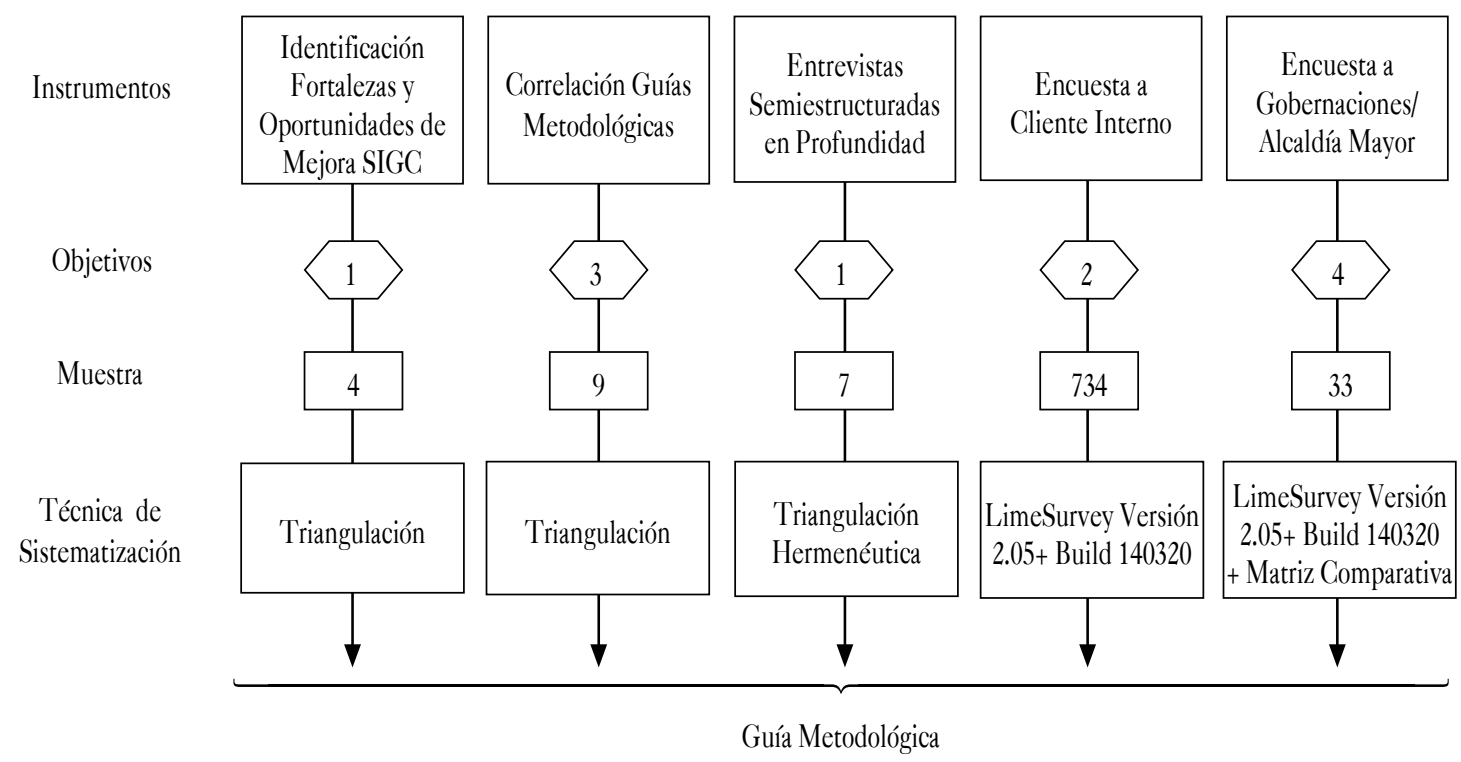

Fuente: elaboración propia. 
En la figura se aprecian por componente los instrumentos, el objetivo al que corresponden, la muestra y la sistematización de la información. En el componente cualitativo los instrumentos definidos se orientan a la obtención de datos para:

- Identificación de fortalezas y oportunidades de mejora del sistema integral de gestión y control de la gobernación, el cual corresponde al objetivo específico 1) identificar las fortalezas y oportunidades de mejora del sistema integral de gestión y control de la Gobernación de Cundinamarca. La muestra determinada corresponde a cuatro documentos revisados del periodo comprendido entre los años 2013 a 2015, a saber: informes de auditorías internas, externas, de control interno y revisión por la dirección, con sistematización por triangulación.

- Correlacionar las guías metodológicas y otros documentos nacionales e internacionales, llevando a cabo una identificación de aspectos comunes, distantes y la correlación con la estructura de alto nivel, el cual corresponde al objetivo específico 3) correlacionar algunas metodologías de implementación de sistemas de gestión existentes en el contexto nacional e internacional. Las guías determinadas corresponden a nueve documentos con sistematización de datos por triangulación, a saber:

1. Guía NTC-ISO 18091:2014, "sistemas de gestión de la calidad, para la aplicación de la norma ISO 9001:2008 en gobiernos locales”.

2. Guía para la integración de los sistemas de gestión UNE66177:2005.

3. Especificación PAS 99:2012 (Miguel, 2013).

4. Anexo SL parte 1, estructura de alto nivel (HLS) para las normas de los sistemas de gestión (SG) (ISO, 2016).
5. Diseño de un sistema de gestión integral gerencia de planta de CVG Minerven (Marchan, 2009).

6. Guía metodológica para la implementación de un Sistema Integrado de Gestión en la empresa Cortiplast S.A. (Flores y Arellano, 2012).

7. Métodos de integración basados en organismos nacionales e internacionales - Departamento Administrativo de la Función Pública.

8. Guía metodológica modelo conceptual y operativo para la implantación de Sistemas Integrados de Gestión - Secretaría de Salud de Cundinamarca (Gobernación de Cundinamarca, 2001).

9. Metodología de implementación del modelo integrado de planeación y gestión.

- Realización de entrevistas semiestructuradas en profundidad, con el fin de determinar la metodología de integración utilizada, percepción del estado del sistema integral de gestión y control de la gobernación, expectativas frente a una guía metodológica, aciertos y dificultades evidenciados durante la implementación, el cual corresponde al objetivo específico 1) identificar las fortalezas y oportunidades de mejora del sistema integral de gestión y control de la Gobernación de Cundinamarca. La entrevista se aplicó al universo el cual corresponde a siete funcionarios de la dirección de desarrollo organizacional, dependencia que lidera la implementación del sistema en la entidad. La sistematización se llevó a cabo por triangulación hermenéutica para obtener mayor precisión en la información obtenida.

En cuanto al componente cuantitativo:

- El cuarto instrumento definido fue la encuesta a clientes internos, para establecer el grado de 
conocimiento, apropiación y la percepción por parte del cliente interno de la gobernación sobre el sistema integral de gestión y control, diseñando para ello un instrumento compuesto por dos secciones, a saber: información general e información específica. La primera para obtener información sobre el proceso en el que se desempeña el cliente interno, el tiempo de vinculación, el conocimiento sobre el sistema integral de gestión y control y su participación, a través de preguntas de selección múltiple. La segunda sección elaborada con escala de Likert para identificar la percepción del cliente interno sobre el sistema integral de gestión y control.

Este instrumento corresponde al objetivo específico 2) evaluar la apropiación y conocimiento del sistema integral de gestión y control por parte del cliente interno en los diferentes procesos y niveles jerárquicos. La muestra determinada corresponde a 734 funcionarios de los diferentes procesos y niveles jerárquicos de la gobernación, contemplando poblaciones de diferentes tiempos de vinculación, procesos y niveles jerárquicos. La sistematización se llevó a cabo a través del software utilizado en la gobernación: LimeSurvey Versión 2.05+ Build 140320, aplicación de software libre y de código abierto.

- El quinto instrumento diseñado fue la encuesta a gobernaciones y a la Alcaldía Mayor de Bogotá, con el fin de identificar en las gobernaciones y alcaldías del país, las metodologías de integración utilizadas, los sistemas implementados, las dificultades en la implementación y las expectativas frente a una guía metodológica. La encuesta consta de doce preguntas de selección múltiple, con opción de respuesta abierta en algunas de ellas.

Este instrumento corresponde al objetivo específico 4) identificar los sistemas implementados en gobernaciones y en la Alcaldía Mayor de Bogotá, metodologías utilizadas, dificultades y expectativas frente a una guía metodológica. El universo a encuestar corresponde a 33 entidades, incluida la Gobernación de Cundinamarca. La sistematización se llevó a cabo a través del software utilizado en la gobernación: LimeSurvey Versión 2.05+ Build 140320 .

Posterior al diseño y elaboración de los instrumentos se llevó a cabo el proceso de validación por parte de expertos, de los instrumentos a aplicar a los funcionarios de la dirección de desarrollo organizacional, a los clientes internos de la gobernación, gobernaciones y la Alcaldía Mayor, con el fin de disponer de instrumentos de medición adecuados con los que se pueda captar la información que se requiere para medir las variables, contenidas en las hipótesis, planteadas en la formulación del proyecto de investigación, por lo cual se procedió a definir el perfil de los expertos para llevar a cabo la validación de los mismos, con el fin de determinar si los instrumentos realmente miden la variable que pretende medir.

La validación del instrumento de entrevista semiestructurada a profundidad, a aplicar a los funcionarios de la dirección de desarrollo organizacional de la Secretaría de la Función Pública de la Gobernación, fue realizada por tres expertos con perfil de posgrado en Psicología del Consumidor, con profesión base en Psicología o en Publicidad, y por parte de la Secretaría de la Función Pública de la Gobernación, quien autorizó la aplicación como representante de la alta dirección para el sistema integral de gestión y control de la gobernación.

Tabla 2. Variables selección expertos validación instrumento. Entrevista cliente interno.

\begin{tabular}{|l|}
\hline \multicolumn{1}{|c|}{ Variables para la selección de expertos } \\
\hline Título de posgrado en Psicología del Consumidor. \\
\hline Profesión base Psicología o Publicidad. \\
\hline
\end{tabular}

Fuente: elaboración propia. 
Por su parte para la validación de las encuestas a cliente interno, gobernaciones y en la Alcaldía Mayor de Bogotá, el perfil de experto incluyó formación con título de posgrado, desarrollo de actividades docentes y experiencia en proyectos en el sector público. Se seleccionaron dieciséis expertos para ser consultados y dos entidades, la facultad de estadística de la Universidad
Santo Tomás y la Fundación Internacional para el Desarrollo de Gobiernos Confiables - FIDEGOC, pretendiendo obtener mínimo tres validaciones y logrando seis en el instrumento a aplicar en gobernaciones y cuatro para el instrumento a aplicar al cliente interno de la gobernación (tabla 3).

Tabla 3. Validación encuesta gobernaciones y Alcaldía Mayor de Bogotá.

\begin{tabular}{|c|c|c|c|c|c|c|c|c|c|c|c|c|c|c|c|c|c|c|c|c|}
\hline \multirow[b]{2}{*}{$\begin{array}{l}\text { VARIABLES PARA } \\
\text { SELECCIÓN } \\
\text { EXPERTOS }\end{array}$} & \multicolumn{20}{|c|}{ EXPERTOS CONSULTADOS } \\
\hline & 密 & 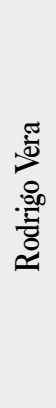 & 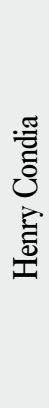 & . & 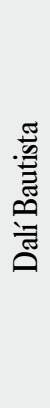 & 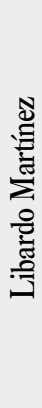 & 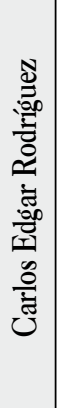 & 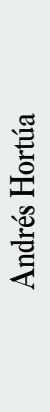 & 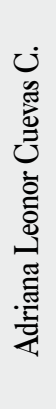 & 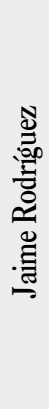 & 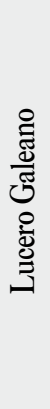 & 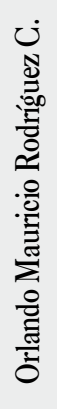 & 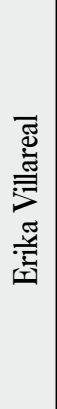 & 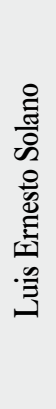 & 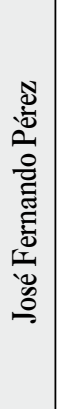 & 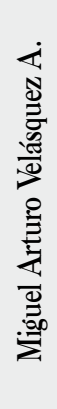 & 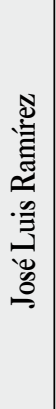 & 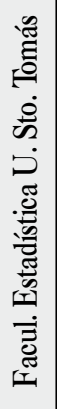 & 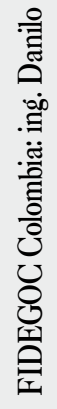 & 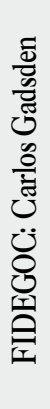 \\
\hline $\begin{array}{l}\text { Título de } \\
\text { posgrado }\end{array}$ & 1 & 1 & 1 & 1 & 1 & 1 & 1 & 1 & 1 & 1 & 1 & 1 & 1 & 1 & 1 & 1 & 1 & & & \\
\hline $\begin{array}{l}\text { Actividades } \\
\text { docentes/ } \\
\text { académicas }\end{array}$ & 1 & 1 & 1 & 1 & 1 & 1 & 1 & 1 & 1 & 1 & 1 & 1 & 1 & 1 & 1 & & 1 & 1 & & \\
\hline $\begin{array}{l}\text { Experiencia en } \\
\text { proyectos de } \\
\text { sector público }\end{array}$ & 1 & 1 & 1 & 1 & 1 & 1 & 1 & 1 & 1 & 1 & 1 & 1 & 1 & 1 & 1 & 1 & & & 1 & 1 \\
\hline Cliente interno & & & & & & & $\mathrm{x}$ & & & $\mathrm{x}$ & & & $\mathrm{x}$ & & & & $\mathrm{x}$ & & & \\
\hline Gobernaciones & $\mathrm{x}$ & & & & & & & & & $x$ & $\mathrm{x}$ & $\mathrm{x}$ & & $x$ & & & & $\mathrm{x}$ & & \\
\hline
\end{tabular}

Fuente: elaboración propia.

\section{Criterios de validez y confiabilidad}

Nivel de confianza $90 \%, 95 \%$ y $99 \%$ y unos errores de 0.02 y 0.03 , donde el nivel de confianza expresa la certeza de que realmente el dato que buscamos esté dentro del margen de error, siendo este el intervalo en el cual se espera encontrar el dato que se quiere medir.

En cuanto a la planeación de los criterios de validez y confiabilidad se definió utilizar la validez de constructo, entendida esta como una variable que tiene lugar dentro de la hipótesis. Y se definió someter a validación, los instrumentos a utilizar.

\section{RESULTADOS Y DISCUSIÓN}

La gobernación cuenta con un sistema integral de gestión y control, integrado por los Sistemas de Desarrollo Administrativo, el Modelo Estándar de Control Interno y el sistema de gestión de la calidad, y está 
certificada con las normas NTC ISO 9001:2008 y NTC GP 1000:2009, sin embargo no contaba con una metodología para la implementación del mismo, a partir de ellos la investigación plantea seis objetivos concretos presentados a continuación, junto con los resultados especificos de cada uno de ellos que permitieron elaborar la guía metodológica para la implementación del SIGG como gran resultado final:

Tabla 4. Resultados alcanzados en función de los objetivos

\begin{tabular}{|c|c|}
\hline OBJETIVOS & RESULTADOS \\
\hline $\begin{array}{l}\text { 1. Identificar las fortalezas y oportunidades de mejora del sistema } \\
\text { integral de gestión y control de la gobernación. }\end{array}$ & $\begin{array}{l}\text { Cualitativo. } \\
\text { Fortalezas y oportunidades de mejora del SIGC, aciertos y desaciertos, } \\
\text { estructura de la guía. }\end{array}$ \\
\hline $\begin{array}{l}\text { 2. Evaluar la apropiación y conocimiento del SIGC por parte del cliente } \\
\text { interno en los diferentes procesos y niveles jerárquicos. }\end{array}$ & $\begin{array}{l}\text { Cuantitativo. } \\
\text { Tiempo de vinculación. } \\
\text { Conocimientos sobre el SIGC. } \\
\text { Roles asumidos en el SIGC. } \\
\text { Participación en actividades. } \\
\text { Percepción sobre el aporte del SIGC. }\end{array}$ \\
\hline $\begin{array}{l}\text { Correlacionar algunas metodologías de implementación de sistemas de } \\
\text { gestión existentes en el contexto nacional e internacional. }\end{array}$ & $\begin{array}{l}\text { Cualitativo. } \\
\text { Características. } \\
\text { Estructura individual. } \\
\text { Correlación con estructura de alto nivel. }\end{array}$ \\
\hline $\begin{array}{l}\text { 3. Identificar sistemas implementados, metodologías, dificultades y } \\
\text { expectativas en gobernaciones y en la Alcaldía Mayor. }\end{array}$ & $\begin{array}{l}\text { Cuantitativo. } \\
\text { Metodología de integración utilizada. } \\
\text { Dificultades en la integración. } \\
\text { Contribución de los sistemas implementados. } \\
\text { Expectativas frente a una guía. }\end{array}$ \\
\hline 4. Validar la guía metodológica. & Validación guía en panel de expertos. \\
\hline 5. Presentar la iniciativa de publicación. & $\begin{array}{l}\text { Presentación de iniciativa de publicación en gobernación y en } \\
\text { convenio USTA-Icontec. }\end{array}$ \\
\hline
\end{tabular}

Fuente: elaboración propia.

En cuanto al primer objetivo, dentro de las fortalezas identificadas están: el compromiso del gobernador, disponer de recursos humanos y financieros e indicadores por proceso alineados con los objetivos del sistema, así como la herramienta de gestión documental, ISOlucion, que tiende a tornarse en oportunidad de mejora por cuanto se confunde con el sistema integral de gestión y control. En tanto que las oportunidades de mejora, a la luz del concepto de Sistema Integral de Gestión (SIG) de Rocha y Karapetrovic (2015), a la fecha el sistema de la gobernación no es visto como una alternativa para vincular nuevos sistemas al existente, se observa desconexión entre el sistema integral de gestión y control y otros obligatorios como: el del sector educación y el obligatorio de garantía de la calidad que aplica al sector salud, el sistema de seguridad y salud en el trabajo y con el sistema de gestión de seguridad de la información, siendo evidente que se cuenta con varios sistemas aislados, que exigen recursos y esfuerzos independientes, por ende se presentan reprocesos. Así mismo se identifica que el sistema está implementado por procesos siendo la gestión institucional por estructura funcional, lo cual puede afectar el desempeño del sistema. 
Como resultante del segundo objetivo se evidencia que el cliente interno desconoce el nivel del mapa de procesos al que pertenece, y en algunos casos el proceso en el que se encuentran, igual sucede con la conformación del sistema, se identifica mayormente el sistema de gestión de la calidad. Adicionalmente se observa baja conceptualización y apropiación de lo que es el sistema integral de gestión y control en todos los niveles jerárquicos y en los diferentes procesos, independiente del tiempo de vinculación a la entidad, viendo así la necesidad de fortalecer la capacitación, la sensibilización y los estímulos para fortalecer la participación y compromiso con el sistema, logrando que sea entendido desde el propósito por cual se decidió implementarlo. Al momento de realizar la investigación se encontró que el conocimiento y apropiación del sistema integral de gestión y control es inversamente proporcional a la forma como lo percibe el mismo cliente interno, debido a que los clientes internos lo perciben en forma positiva, en promedio el $76.95 \%$, pero no lo conocen y no entienden que es frente a las diferentes variables encuestadas, a saber: aporte del SIGC al cumplimiento de metas del Plan Departamental de Desarrollo, conocimiento del sistema integral y sus beneficios, identificación de necesidades y expectativas a través del SIGC.

En cuanto a la metodología utilizada por otras gobernaciones, gobernaciones y la Alcaldía Mayor que respondieron completamente la encuesta (Antioquia, Risaralda, Guaviare, Valle del Cauca, Arauca y Meta), se identifica que utilizaron para la implementación la metodología del departamento administrativo de la función pública. Corroborando con ello la hipótesis de que las gobernaciones del país y la Alcaldía Mayor han utilizado la metodología del DAPF para la implementación de sus sistemas de gestión.

Como resultado final de la investigación se diseñó la guía metodológica para la implementación del sistema integral de gestión y control de la Gobernación de Cundinamarca que responde a los requerimientos propios de la entidad; es sencilla, clara, de fácil lectura, propia para la Gobernación de Cundinamarca, respondiendo al por qué, al cómo y al quehacer para la implementación del sistema integral de gestión y control. La guía fue validada en dos momentos, el primero de ellos mediante panel de expertos de la Secretaría de Salud; el segundo momento con participación de la Secretaría de la Función Pública y la directora de Desarrollo Organizacional, presentándose como un documento apropiado para ser implementado en la gobernación al responder al problema planteado disponiendo de una metodología para la implementación de múltiples sistemas de gestión en uno solo, y al permitir un mejor uso de los recursos, objetivos compartidos y procesos claramente interrelacionados.

Así mismo la guía metodológica diseñada aborda las principales dificultades identificadas en las gobernaciones y la Alcaldía Mayor para la implementación de sus sistemas de gestión en cuanto a la falta de capacitación, de compromiso y de continuidad de los servidores públicos con los sistemas de gestión, el escaso compromiso de la alta dirección, la falta de políticas institucionales, la desarticulación de objetivos y metas del Plan Departamental de Desarrollo con los sistemas de gestión, la inexistencia de gestores en todos los niveles de la entidad, la baja credibilidad en el aporte de los sistemas de gestión al logro de los objetivos del Plan Departamental de Desarrollo y la falta de orientación metodológica para la integración de los sistemas de gestión, y resuelve una de las mayores expectativas de las gobernaciones y la Alcaldía Mayor con relación a una metodología al permitir articular los sistemas de gestión con los planes de desarrollo, y fomentar así el compromiso del cliente interno en todos los niveles. La estructura del documento consta de: preliminares (introducción, objetivo y alcance); tres capítulos, a saber: El sistema integral de gestión y control - SIGC, 
metodología de implementación, Material de apoyo para la implementación y un anexo denominado: La liga de la gestión integral: porque el cuento de la gestión integral SÍ es contigo, el cual contiene material de apoyo técnico presentado en forma de historieta de superhéroes.

Se presentó la iniciativa de publicación de la guía en la Gobernación de Cundinamarca, para la generación de conocimiento y la apropiación social del mismo, es aplicable en el sector público y contiene material lúdico dirigido al cliente interno facilitando la apropiación del sistema.

\section{CONCLUSIONES}

Se diseñó una guía metodológica para la implementación del sistema integral de gestión y control de la Gobernación de Cundinamarca sustentada en la información recolectada, analizada y correlacionada, aportando a la entidad por cuanto no se disponía de una metodología específica, y generando valor al ser posible a través de ella implementar todos los sistemas obligatorios y voluntarios que se requieran.

Las principales fortalezas del sistema implementado a la fecha son: contar con el compromiso del gobernador, la asignación de recursos humanos y financieros, la adecuada alineación de los indicadores por proceso con los objetivos del sistema, la certificación del sistema de gestión de calidad con las normas NTC ISO 9001:2008 y NTC GP 1000:2009, la herramienta de gestión documental ISOlucion y la oportunidad de mejora del sistema integral de gestión y control es la integración de otros sistemas obligatorios como el del sector educativo, el del sector salud, el de seguridad y salud en el trabajo y otro voluntarios plasmados dentro del Plan Departamental de Desarrollo. Así como mejorar la comunicación interna, el conocimiento y apropiación del sistema por parte del cliente interno ya que lo percibe favorable y beneficioso, lo cual es inversamente proporcional a su conocimiento.

Para fortalecer el conocimiento y apropiación del sistema por parte de los clientes internos, así como la comunicación, se requiere determinar estrategias apropiadas por lo cual la guía incluye un documento lúdico en forma de cómic, el cual fue presentado para ser adoptado por la gobernación para una publicación periódica.

Las gobernaciones y la Alcaldía Mayor que respondieron completamente la encuesta (Antioquia, Risaralda, Guaviare, Valle del Cauca, Arauca y Meta) utilizaron para la implementación de sus sistemas de gestión la metodología del Departamento Administrativo de la Función Pública. Corroborando con ello la hipótesis de que las gobernaciones del país y la Alcaldía Mayor han utilizado la metodología del DAPF para la implementación de sus sistemas de gestión.

La guía metodológica contempla el abordaje de las principales dificultades identificadas en las gobernaciones y la Alcaldía Mayor para la implementación de sus sistemas de gestión en cuanto a la falta de capacitación, de compromiso y de continuidad de los servidores públicos con los sistemas de gestión, el escaso compromiso de la alta dirección, la falta de políticas institucionales, la desarticulación de objetivos y metas del Plan Departamental de Desarrollo con los sistemas de gestión, la inexistencia de gestores en todos los niveles de la entidad, la baja credibilidad en el aporte de los sistemas de gestión al logro de los objetivos del Plan Departamental de Desarrollo y la falta de orientación metodológica para la integración de los sistemas de gestión.

La guía metodológica elaborada fue revisada y ajustada en lo pertinente de acuerdo con la validación, realizada en dos momentos: el primero mediante un panel de expertos de la Secretaría de Salud; el segundo con la 
representante de la alta dirección y la directora del área que lidera la implementación del sistema, de tal manera que se presenta como un documento apropiado para ser implementado en la gobernación.

Se presentó la iniciativa de publicación de la Guía en la Gobernación de Cundinamarca, soportada en la aprobación inicial del proyecto de investigación, la cual se encuentra en proceso de aprobación.

Con esta investigación se da respuesta al problema que la originó, corroborando las hipótesis de que la gobernación no disponía de una metodología para la implementación del sistema, y que la metodología más utilizada en otras entidades del mismo orden es la del Departamento Administrativo de la Función Pública, por otra parte, frente a la tercera hipótesis, el resultado arroja que no es directamente proporcional el conocimiento/apropiación del cliente interno versus la percepción sobre el sistema integral de gestión y control.

Como resultado de la investigación se genera conocimiento al disponer de un nuevo documento metodológico para la implementación de sistemas integrales de gestión.

\section{AGRADECIMIENTOS}

A la Gobernación de Cundinamarca, a la Federación de Departamentos, a las gobernaciones y a la Alcaldía mayor de Bogotá por su participación.

\section{REFERENCIAS}

Capra, F. (1998). La trama de la vida. Una nueva perspectiva de los seres vivos. ISBN 84-339-0554-6. Barcelona: Editorial Anagrama. Colección argumentos.
Congreso de la República de Colombia. (29 de diciembre de 1998). Por la cual se dictan normas sobre la organización y funcionamiento de las entidades del orden nacional, se expiden las disposiciones, principios y reglas generales para el ejercicio de las atribuciones previstas en los numerales 15 y 16 del artículo 189 de la Constitución Politica y se dictan otras disposiciones. [Ley 489 de 1998]. DO: 43.464.

Congreso de la República de Colombia. (30 de diciembre de 2003). Por la cual se crea el sistema de gestión de la calidad en la rama ejecutiva del poder público y en otras entidades prestadoras de servicios. [Ley 872 de 2003]. DO: 45.418.

Deming, W. (1989). Calidad, productividad y competitividad: la salida de la crisis. Madrid, España: Ediciones Díaz de Santos S. A.

Departamento Administrativo de la Función Pública. (17 de marzo de 2005). Por el cual se establece el sistema de nomenclatura y clasificación y de funciones y requisitos generales de los empleos de las entidades territoriales que se regulan por las disposiciones de la Ley 909 de 2004. [Decreto 785 de 2005]. DO: 45.855.

Departamento Administrativo de la Función Pública. (21 de mayo de 2014). Por el cual se actuali氵a el Modelo Estándar de Control Interno - MECI. [Decreto 943]. DO: 49.158.

Flores, G., y Arellano, L. (2012). Modelo de un Sistema Integrado de Gestión. Tesis de maestría. Guayaquil: Universidad Politécnica Salesiana.

Fraguela, F., Carral, C., Iglesias, G., Castro, A., y Rodríguez, J. (2011). La integración de los sistemas de gestión. Necesidad de una nueva cultura empresarial, 78(7), 44-49. Recuperado de http://revistas.unal. edu.co/index.php/dyna/article/view/25761/26188 
Gobernación de Cundinamarca. (1 de abril de 2015). Por el cual se establece la estructura de la Administración Pública Departamental, se define la organiaación interna y las funciones de las dependencias del sector central de la administración puiblica de Cundinamarca y se dictan otras disposiciones. Sector Central: Despacho del Gobernador, Jefatura de Gabinete, Secretarías de Despacho, Oficinas, Consejos y Comités Superiores de la Administración Pública Departamental. [Decreto ordenanzal 066]. Gaceta de Cundinamarca: 15.172.

Gobernación de Cundinamarca. (2001). Guía Metodológica Modelo Conceptual y Operativo para la Implementación del Sistema Integrado de Gestión en 37 Hospitales de la Red Pública y en la Secretaría de Salud de Cundinamarca. Secretaría de Salud.

Gobernación de Cundinamarca. (22 de octubre de 2008). Por el cual se modifica el Estatuto Básico de la Administración Departamental de Cundinamarca modificado mediante el Decreto 0025 de 2005 y se dictan otras disposiciones. [Decreto ordenanzal 258]. Gaceta de Cundinamarca: 14.894.

Hernández, R., Fernández, C., y Baptista P. (2014). Metodología de la Investigación. (6. ${ }^{\mathrm{a}}$ ed.), pp. $532-$ 554. México: McGraw-Hill.

Instituto Colombiano de Normas Técnicas y Certificación. (2008). NTC-ISO 9001:2008. Sistemas de gestión de la calidad. Requisitos. Norma Técnica de Calidad. Bogotá: Icontec.
ISO. (27 de julio de 2016). Consolidated ISO supplement - procedured especific to ISO. Switzerland. Recuperado de https://isotc.iso.org/livelink/ livelink/fetch/2000/2122/4230450/4230452/ ISO_IEG_Directives_Part_1_and_Consolida ted_ISO_Supplement_\%2D_2016_\%287th_edi tion\%29_\%2D_PDF.pdf?nodeid=17668772\&ve rnum $=-2$

Karapetrovic, S., y Willborn, W. (1998) Integración de la calidad y sistemas de gestión ambiental. The TQM Magasine, 10(3), 204-213. Recuperado de https://dx.doi.org/10.1108/09544789810214800

Marchan, F. (2009). Diseño de un sistema de gestión integral. Tesis de maestría, Universidad Nacional Experimental Politécnica "Antonio José de Sucre". Recuperado de https:/es.scribd.com/document/307269593/diseno-sistema-gestion-inte gral-doc

Miguel, L. (2013). PAS 99. Especificación de los requisitos comunes del sistema de gestión como marco para la integración. Revista Calidad. La gestión de la innovación, (1), 10-14. Recuperado de https://www.aec.es/c/document_library/get_ file? uuid=d4f00264-ee74-4abd-b1c8-4e48fccd 6836\&groupId=10128

Rocha, M. y Karapetrovic, S. (2008). Creando un Sistema Integral de Gestión - necesidades de la evolución de ISO 9001 y otros estándares de gestión. Recuperado de http://148.204.210.204/revistaupiicsa/46/46-2.pdf 